\title{
Prophylactic anticoagulation in hospitalized patients with COVID-19: less is better
}

\section{Anticoagulación profiláctica en pacientes hospitalizados durante la COVID-19: menos es mejor}

\begin{abstract}
Mateo Porres-Aguilar ${ }^{1 *}$, Orlando R. Pérez-Nieto², Javier E. Anaya-Ayala³, Eder I. Zamarrón-López Thierry Hernández-Gilsoul${ }^{5}$ and Moisés Aurón ${ }^{6}$

${ }^{1}$ Department of Internal Medicine, Division of Hospital Medicine, Texas Tech University Health Sciences Center, EI Paso, Texas, USA; ${ }^{2}$ Department of Intensive Care and Critical Medicine, Hospital San Juan del Río, Querétaro, Mexico; ${ }^{3}$ Department of Surgery, Vascular Surgery and Endovascular Therapy Section; Instituto Nacional de Ciencias Médicas y Nutrición Salvador Zubirán, Mexico City, Mexico; ${ }^{4}$ Intensive Care and Critical Medicine Unit, Hospital CEMAIN-Tampico and IMSS Hospital no. 6, Ciudad Madero, Tamaulipas, Mexico; ${ }^{5}$ Department of Emergency Medicine, Instituto Nacional de Ciencias Médicas y Nutrición Salvador Zubirán, Mexico City, Mexico; ${ }^{6}$ Department of Hospital Medicine and Pediatric Hospital Medicine, Cleveland Clinic, Cleveland, Ohio, USA
\end{abstract}

We read with interest the article published by Ignacio-lbarra et al. 1 . The authors propose therapeutic anticoagulation with heparin and/or rivaroxaban for hospitalized patients with coronavirus disease 2019 (COVID-19) without clinical improvement or with D-dimer levels $>3,000 \mathrm{ng} / \mathrm{mL}$. We want to share the following: the ACTION trial, which compared anticoagulant treatment at therapeutic doses vs. prophylaxis in 615 patients, was recently published ${ }^{2}$. The primary endpoint was a hierarchical analysis of mortality, hospitalization time and oxygen supplementation at 30 days. Of the patients with the therapeutic anticoagulation strategy, $90 \%$ received oral rivaroxaban at $20 \mathrm{mg} /$ day. No significant differences were found between both groups regarding the primary composite endpoint: therapeutic strategy $34.8 \%$ vs. prophylactic strategy $41.3 \%$ (odds ratio [OR]: 0.86; 95\% confidence interval [Cl]: $0.59-1.22 ; \mathrm{p}=0.40$ ). However, there was a significant difference between both groups with regard to the safety endpoints, major or clinically relevant hemorrhage: therapeutic strategy $8 \%$ vs. prophylactic strategy 2\% (OR: 3.6; 95\% Cl: 1.61-8.27, $p=0.001)^{2}$. These results show that an anticoagulation strategy with a therapeutic dose did not confer benefit in comparison with a conventional thromboprophylaxis strategy².

We consider that caution should be maintained in COVID-19 thromboprophylaxis strategies due to a high risk of adverse events, which may outweigh their benefit in the context of difficult clinical scenarios.
Avoiding said strategies based only on D-dimer elevated levels or lack of clinical improvement is suggested. Therapeutic anticoagulation remains reserved for duly diagnosed acute thrombotic events. Upcoming publication of the results of multiplatform, adaptive, prospective, randomized trials (REMA-CAP, ATTACC and ACTIV-4a) will provide more solid evidence on thromboprophylactic strategies for these patients ${ }^{3}$.

\section{Funding}

The authors declare not having received any funding.

\section{Conflict of interests}

The authors declare that they have no conflicts of interest.

\section{References}

1. Ignacio-Ibarra G, García-Lee MT, González-Ávila Al, García-Chávez J, Guzmán-Chores L, Cruz CL, et al. Propuesta para el manejo de la coagulopatía asociada a COVID-19 en adultos. Gac Med Mex. 2021;157:209-14.

2. Lopes RD, de Barros e Silva PGM, Furtado RHM, Macedo AVS, Bronhara B, Damiani LP, et al. Therapeutic versus prophylactic anticoagulation for patients admitted to hospital with COVID-19 and elevated D-dimer concentration (ACTION): an open-label, multicentre, randomised, controlled trial. Lancet. 2021;397:2253-63.

3. Porres-Aguilar M, Pérez-Nieto OR, Zamarrón-López Él, Ñamendys-Silva SA. Thromboprophylaxis strategies for COVID-19 patients. Arch Med Res. 2021; Jul 5: S0188-4409(21)00146-6. doi: 10.1016/j.arcmed.2021.06.011. Online ahead of print. 